\title{
RG WIN 2015. APLICACIÓN PARA EL DISEÑO Y DIMENSIONADO DE REDES DE RIEGO A PRESIÓN EN ENTORNO EXCEL INTEGRANDO PARÁMETROS AGRONÓMICOS, ENERGÉTICOS E HIDRÁULICOS
}

\author{
Arviza Valverde, Jaime (1). Balbastre Peralta, Ibán (2), Palau Estevan, Carmen Virginia (3) \\ ${ }^{1}$ PDI. Universitat Politècnica de València. Dpto. Ingeniería Rural. Jarviza@agf.upv.es \\ ${ }^{2}$ PDI. Universitat Politècnica de València. Dpto. Ingeniería Rural. ibbalpe@agf.upv.es \\ 3 PDI. Universitat Politècnica de València. Dpto. Ingeniería Rural. virpaes@agf.upv.es
}

\section{Resumen}

En la comunicación se presentan los resultados del desarrollo de una aplicación informática en entorno Excel para el diseño y dimensionado de redes de riego a presión.

La aplicación pretende integrar las distintas fases que suponen el diseño de una red de riego a presión teniendo en consideración la información agroclimática para la determinación de los parámetros de riego que condicionan el funcionamiento del sistema. Asimismo se vinculan los cultivos y sus necesidades hídricas con las parcelas que abastecen los hidrantes de la red.

La aplicación también permite el dimensionado de subunidades y su incorporación como datos al dimensionado de la red. Permite gestionar fácilmente las bases de datos de materiales, así como el tratamiento de resultados y su exportación para posterior análisis con aplicaciones como Epanet 2.0.

La aplicación permite importar redes generadas desde Epanet, o introducir la información desde la propia interfaz. Teniendo otra serie de prestaciones que se indicarán en el trabajo.

La aplicación desarrollada pretende ser de utilidad tanto en el ámbito académico como en el profesional siendo además la misma de difusión libre.

\footnotetext{
Abstract

In this paper the results of the development of a computer application in Excel using Visual Basic for application (VBA) for design and sizing of pressurized irrigation networks are presented.

The application aims to integrate the various phases involving the design of an irrigation pressure network climatic data are considering the information for determining the irrigation parameters that influence the system operation. Crops features and their water needs to plots that supply network hydrants are linked.

The application also allows the sizing of subunits and its incorporation as a data network dimensioning. Easily manage databases of materials and processing and export results for further analysis with applications such as Epanet.

The application allows you to import generated from Epanet networks, or enter the information from the interface itself. Having another set of features that are indicated in the workplace.
} 
The developed application intended to be useful both in academia and in the professional being also the same free diffusion.

\section{Introducción. Objetivos}

El departamento de Ingeniería Rural de la Universitat Politècnica de València (UPV) desarrolló una aplicación para el dimensionado hidráulico de redes de riego a presión (RG) en el año 1991. De la misma fueron desarrollándose diversas versiones, incluyendo más prestaciones y potencia de cálculo. Esta aplicación fue ampliamente utilizada tanto en el ámbito académico como por profesionales en proyectos de modernización de regadíos.

Los avances tecnológicos y sobre todo en el campo de la informática han exigido adaptar dicha aplicación a un entorno actualizado y de difusión general. Se optó por aprovechar todo el potencial de Microsoft Excel pues es una aplicación de uso general conocida y utilizada por una gran mayoría de usuarios tanto en el ámbito académico como técnico y profesional.

Bajo estas premisas se ha pretendido desarrollar una aplicación lo más versátil y potente posible que además sea fácil y cómoda de utilizar y permita la transferencia de información desde otras aplicaciones utilizadas en el diseño, análisis y modelización de redes de riego a presión.

Por otra parte se han integrado en la aplicación el tratamiento de la mayor parte de la información que permite abordar el diseño con un enfoque más global, incluyendo la determinación de parámetros de riego, características de cultivos, parcelas, tipos de hidrantes, dimensionado de subunidades y tratamiento de la información agroclimática.

En lo que se refiere al dimensionado de la red se han utilizado criterios de optimización técnico económica mejorando los algoritmos de cálculo y teniendo en cuenta la actual estructura de la tarificación eléctrica cuya influencia sobre la función de costes cada vez es mayor.

\section{Materiales y métodos.}

\subsection{Desarrollo de la aplicación}

La aplicación se ha desarrollado en Excel utilizando la potencialidad del lenguaje de programación Visual Basic for Applications (VBA).

Se optó por utilizar Excel como base de la aplicación pues es una herramienta informática de uso general, con difusión mundial y totalmente reconocida. Además supone una aplicación de uso habitual tanto para técnicos, estudiantes como investigadores en el ámbito de las ingenierías, por lo que el entorno resulta conocido y fácil de manejar. Además la estructura de datos en tablas resulta idónea tanto para definir la configuración y topología de la red, como para organizar la información relativa a cultivos, parcelas, materiales y condiciones de funcionamiento de la red.

Se ha optado por el desarrollo de un Complemento o Add-Ins en Excel para el funcionamiento de la aplicación. Dicho complemento puede ser instalado de forma sencilla por cada usuario y accediendo a Complementos en el menú principal se accede al Menú conceptual de la aplicación RGWIN2015 (RGW). 
Las tablas que aparecen en las distintas hojas del libro abierto son accesibles desde los diferentes menús desplegables y la introducción de datos puede realizarse directamente en las hojas o a través de formularios específicamente diseñados a tal efecto.

Desde el menú desplegable "Datos Red" se accede a la opción "Datos Generales" donde se definen las características específicas de la red (Alimentación, Material, Criterio de dimensionado, Parámetros financieros, parámetros energéticos y tipo de red)

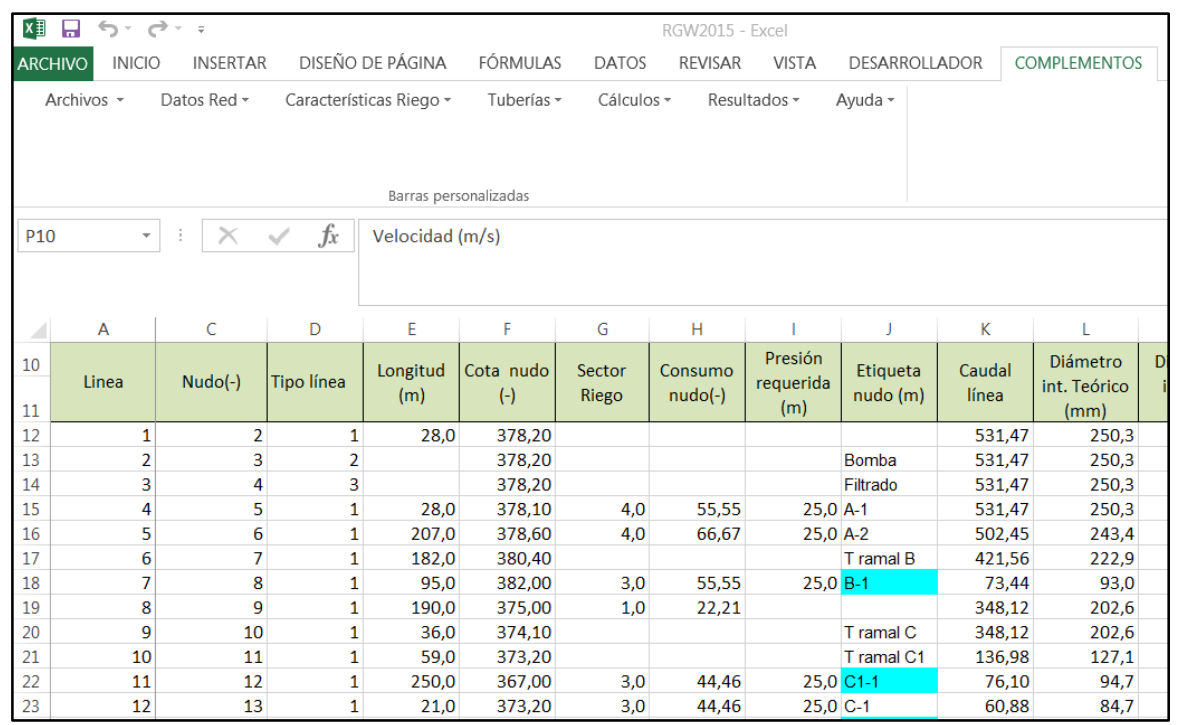

Figura 1: Menú de opciones de la aplicación accesible desde Complementos.

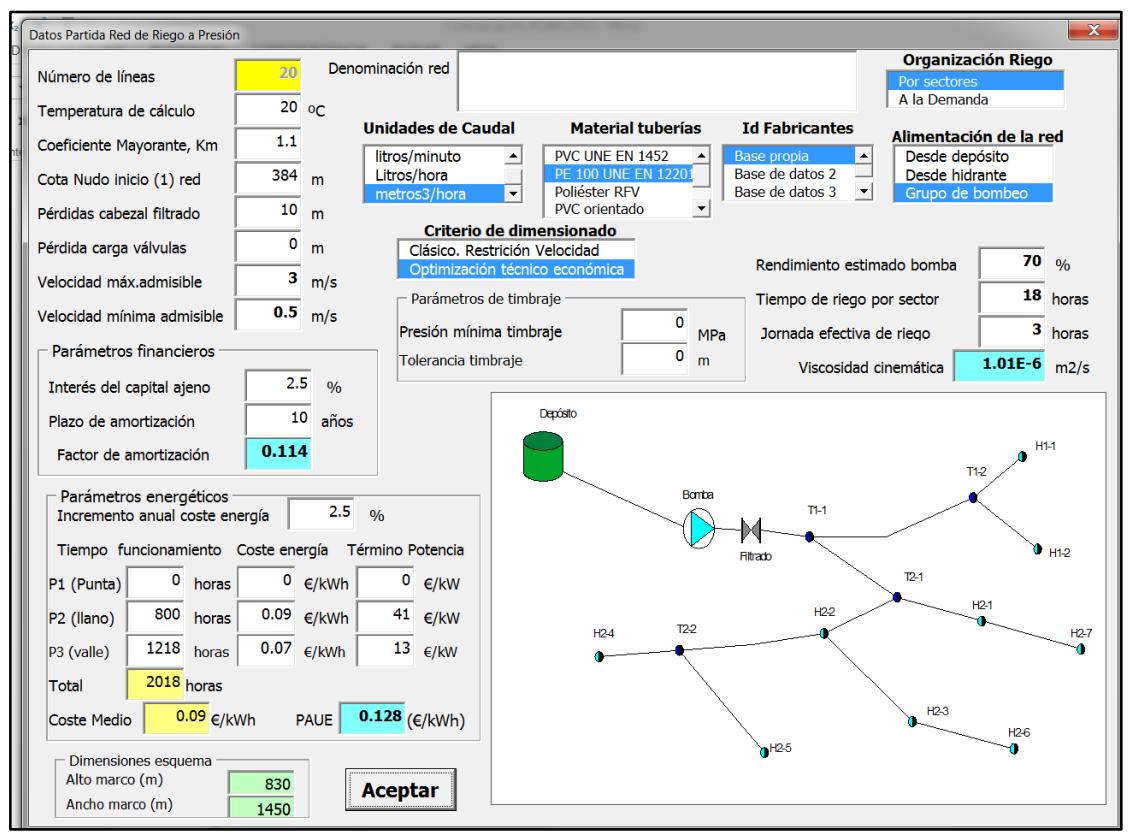

Figura 2: Formulario de datos generales

La red puede ser importada desde la aplicación Epanet ver 2.0, aprovechando en ese caso el entorno gráfico de la misma para definir la topología y configuración de la red.

A su vez se ha previsto la posibilidad de trabajar con esquemas o gráficos con la configuración de la red que facilite la introducción de datos. La numeración de los nudos conectores de cada una de las líneas de la red no tiene que seguir ningún orden o 


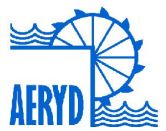

secuencia lo que facilita la identificación de estos y la modificación de datos una vez introducidos.

En cualquier caso se han desarrollado distintas rutinas de captura de errores que permiten detectar previo al cálculo de caudales. El formulario con el esquema de la red (Figura 5) puede estar abierto mientras se introducen los datos en la hoja correspondiente lo que facilita la comprobación de posibles errores. A continuación se muestran dos ejemplos de captura de error en la configuración de la red.

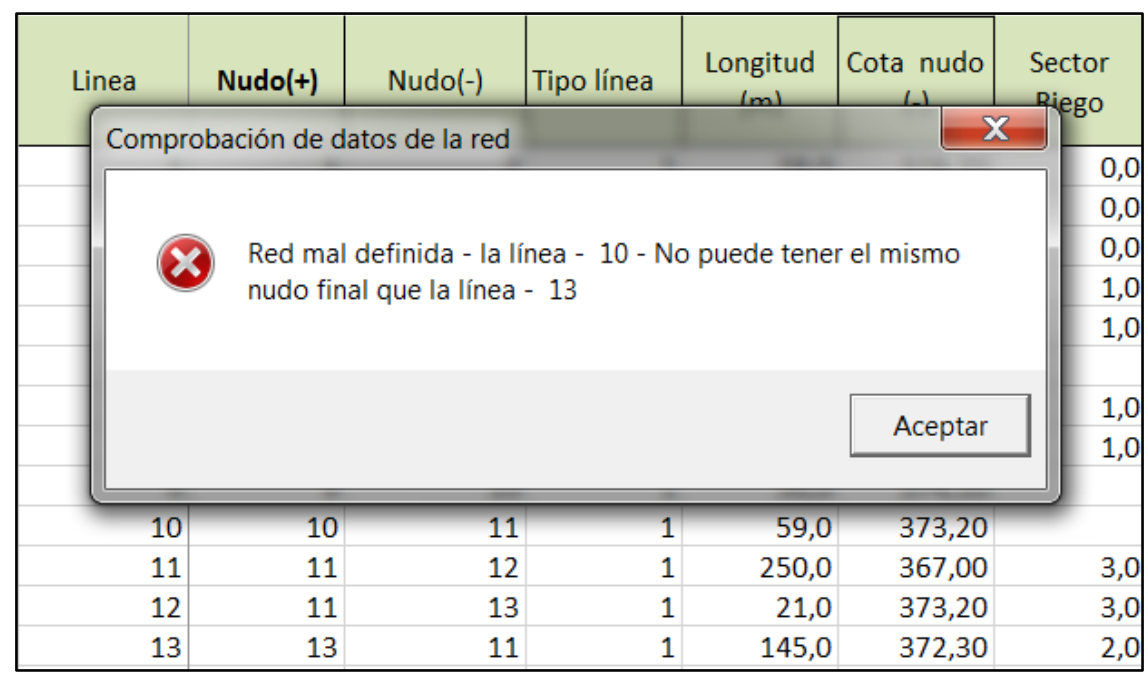

Figura 3

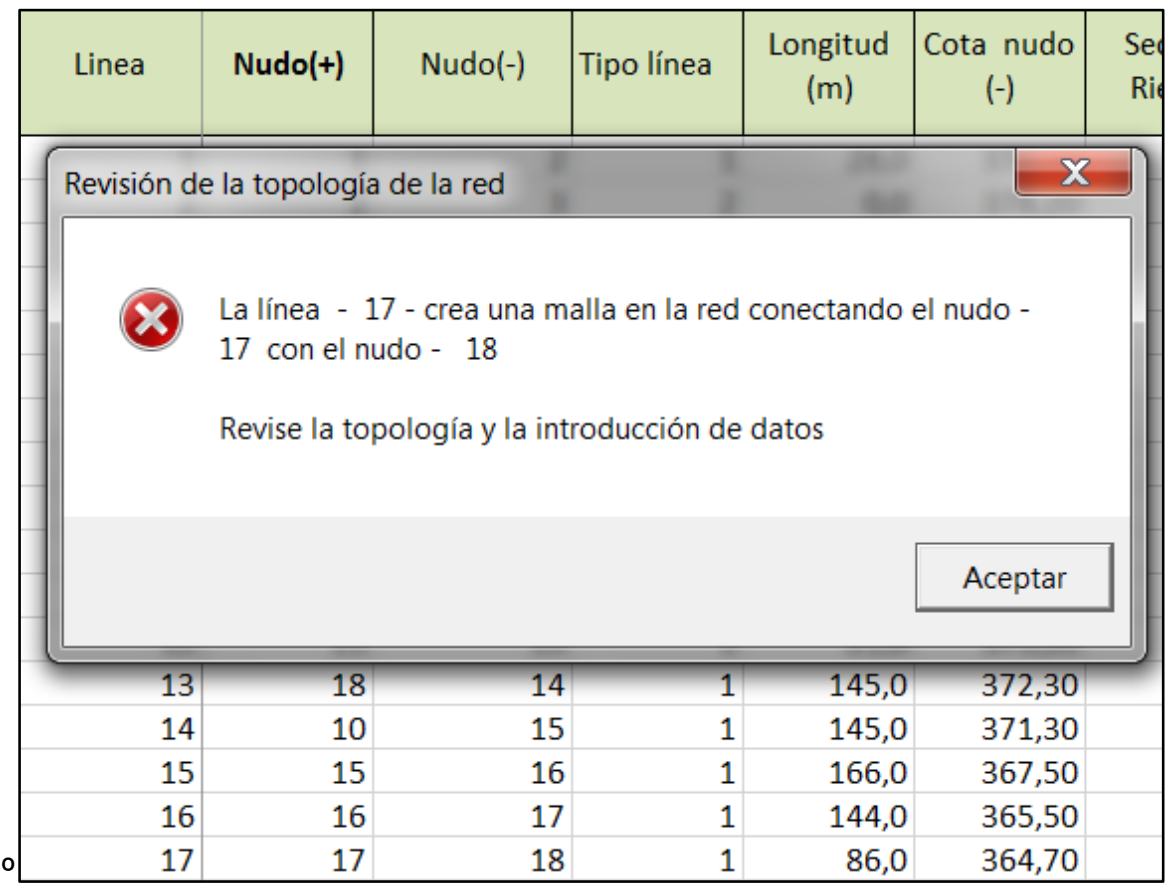

Figura 4 


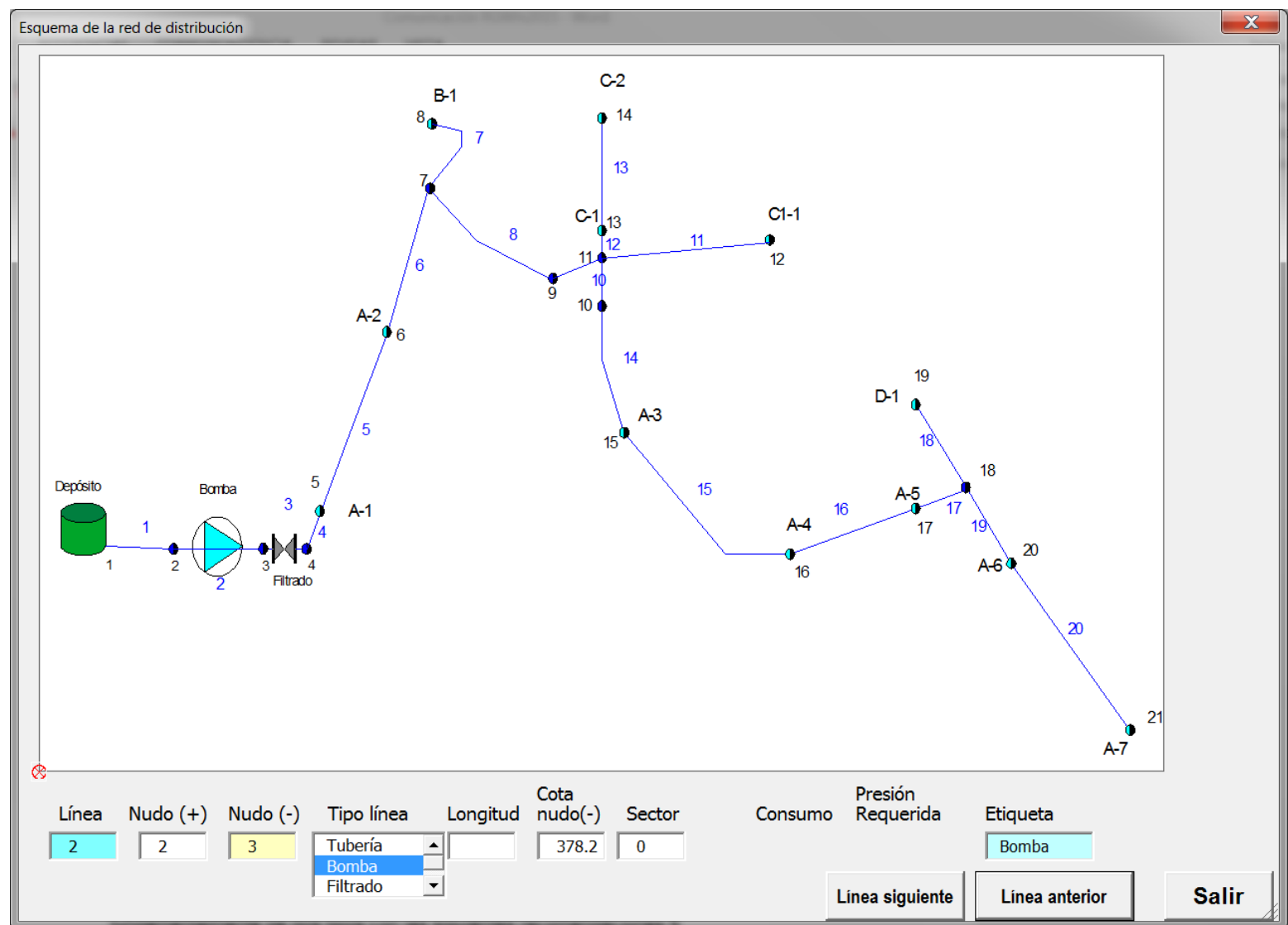

Figura 5: Esquema escalado de la red y formulario para definir la topología y características de la red

Desde el menú "Características de Riego" se accede a dos aplicaciones complementarias y vinculadas en el proceso de diseño de una red de riego:

- Diseño Agronómico RL.xlsm : Cálculo de necesidades hídricas de cultivos en sistemas de riego a presión.

- DimSub.xlsm: Dimensionado y cálculo de subunidades de riego localizado.

Los caudales circulantes por las líneas que componen la red son función de la organización del funcionamiento de la red, pudiendo ser ésta:

- Por turnos o sectores

- A la demanda

En el primer caso los consumos en nudos pueden ser introducidos directamente en la hoja "Cálculos" en la columna correspondiente o vincular el nudo aguas abajo de la línea a un hidrante que alimente a unas parcelas de unas determinadas dimensiones vinculadas a cultivos y condiciones de funcionamiento función dadas. 


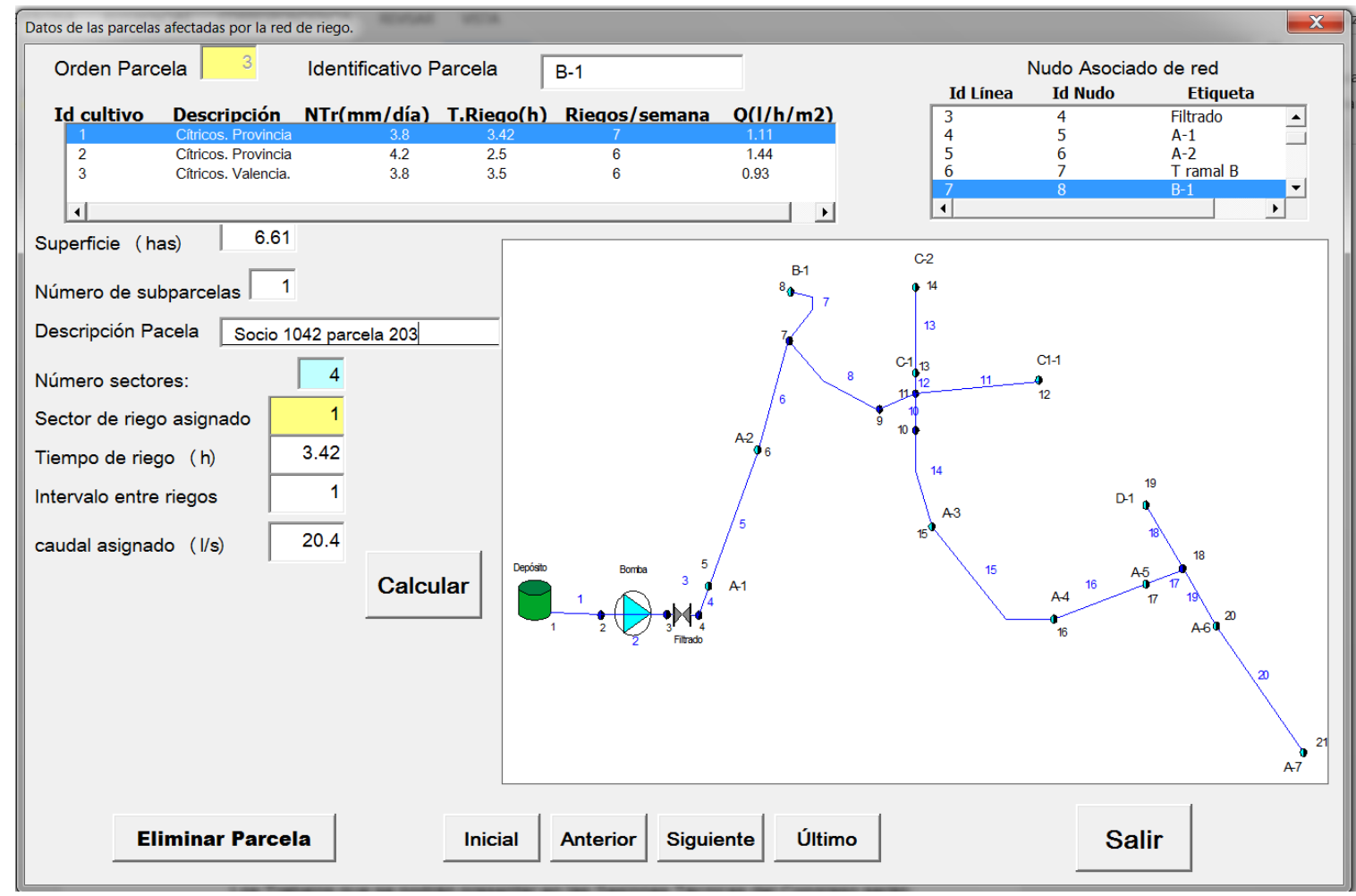

Figura 6: Formulario de parcelas vinculadas a un cultivo y un nudo de la red

\subsection{Criterios de diseño y dimensionado de la red}

Una vez calculados los caudales circulantes por tramo el dimensionado de la red se realiza mediante técnicas de optimización técnico financiera.

En particular RGW utiliza el conocido método de la Serie Económica. Respecto a versiones anteriores los algoritmos de cálculo introducen mejoras en lo que se refiere a:

- Definición de la serie más desfavorable o camino crítico y la descomposición de la red en series de tuberías.

- La función de costes de instalación se ajusta al rango de caudales y velocidades en el dimensionado de cada una de las series.

- Introducción en la función de costes energéticos $\left(\mathrm{K}_{1}\right)$ tanto los términos de energía como de potencia para los distintos periodos contemplados en la tarifa.

- Cálculo del PAUE (Pago anual unificado equivalente) relativo al coste específico de la energía considerando tanto el interés del capital ajeno ( $r$ ) como el incremento anual del coste de la energía $\left(\Delta C_{E}\right)$.

$$
\begin{aligned}
& p=\frac{\sum_{i=1}^{n p} T_{p_{i}}+\sum_{i=1}^{n p}\left(T_{E_{i}} \times T_{i}\right)}{\sum_{i=1}^{n p} T_{i}} \\
& P A U E=\sum_{i=1}^{t}\left[\frac{(1+r)^{t} \cdot r}{(1+r)^{r}-1} \cdot \frac{\left(1+\Delta C_{E}\right)^{t}}{(1+r)^{t}}\right] \cdot p=\alpha \cdot \beta \cdot p
\end{aligned}
$$


Siendo:

$$
K_{1}=\frac{\gamma \cdot Q_{M} \cdot P A U E}{\eta_{e s t}}
$$

(np): Número de periodos de la tarifa eléctrica a contratar. $\left(T_{p_{i}}\right)$ : Término de potencia para cada periodo. $\left(T_{E_{i}}\right)$ : Término de energía para cada periodo. $\left(T_{i}\right)$ : Funcionamiento en horas de la red en cada periodo. (t): Plazo de amortización de la inversión. $\left(Q_{M}\right)$ : Caudal medio en origen con organización del riego a la demanda o caudal máximo en origen para todos los sectores o turnos. $\left(\eta_{e s t}\right)$ : Rendimiento estimado del grupo de bombeo.

\section{Resultados}

Para ilustrar el funcionamiento de la aplicación se ha abordado el diseño y dimensionado de una red.

Se trata de un sector que abastece 12 hidrantes multi usuario y 73 parcelas con una superficie total de 47,7 has.

Las necesidades hídricas de los cultivos han sido calculadas previamente mediante la aplicación "Diseño Agrónomico RL", así como el caudal requerido por unidad de superficie, tiempo e intervalo entre riegos para el periodo de máximos requerimientos y para el resto de la temporada.

La tarifa a contratar en la 3.1.a, habiendo cumplimentado el funcionamiento previsto en cada periodo horario en el formulario de la Figura 2.

El dimensionado se efectúa en dos fases (Cálculo de caudales y Calcular Red)

Una vez efectuado el dimensionado con éxito aparece un formulario con el resumen de resultados (Figura 7). Saliendo del formulario los resultados se listan en la hoja activa (Figura 8). En la columna correspondiente a la presión resultante se marcan en verde aquellas líneas cuyo nudo final es un hidrante o nudo de consumo. El déficit de presión indica para cada nudo la diferencia entre la presión requerida y la resultante.

$$
\text { Déficit }_{i}=\frac{P_{r e q_{i}}}{\gamma}-\frac{P_{(\text {resul tante })_{i}}}{\gamma}
$$

En el caso de requerir bomba en el nudo más desfavorable éste será nulo indicándose en color naranja la correspondiente línea. En la columna correspondiente a Nudo(-) se indica en color el camino crítico o serie más desfavorable. Por último en la siguiente columna aparecen las etiquetas identificativas. Aquellos nudos extremos de la red aparecen en color cian.

En cualquier caso si la red ha sido importada desde Epanet o se han definido las coordenadas de los nudos sobre el esquema escalado (Figura 5) es mucho más intuitivo y sencillo de interpretar los resultados con la opción "Ver Resultados en Esquema". 
XXXIII Congreso Nacional de Riegos

Universitat Politècnica de València, Valencia 2015

DOI:http://dx.doi.org/10.4995/CNRiegos.2015.1461

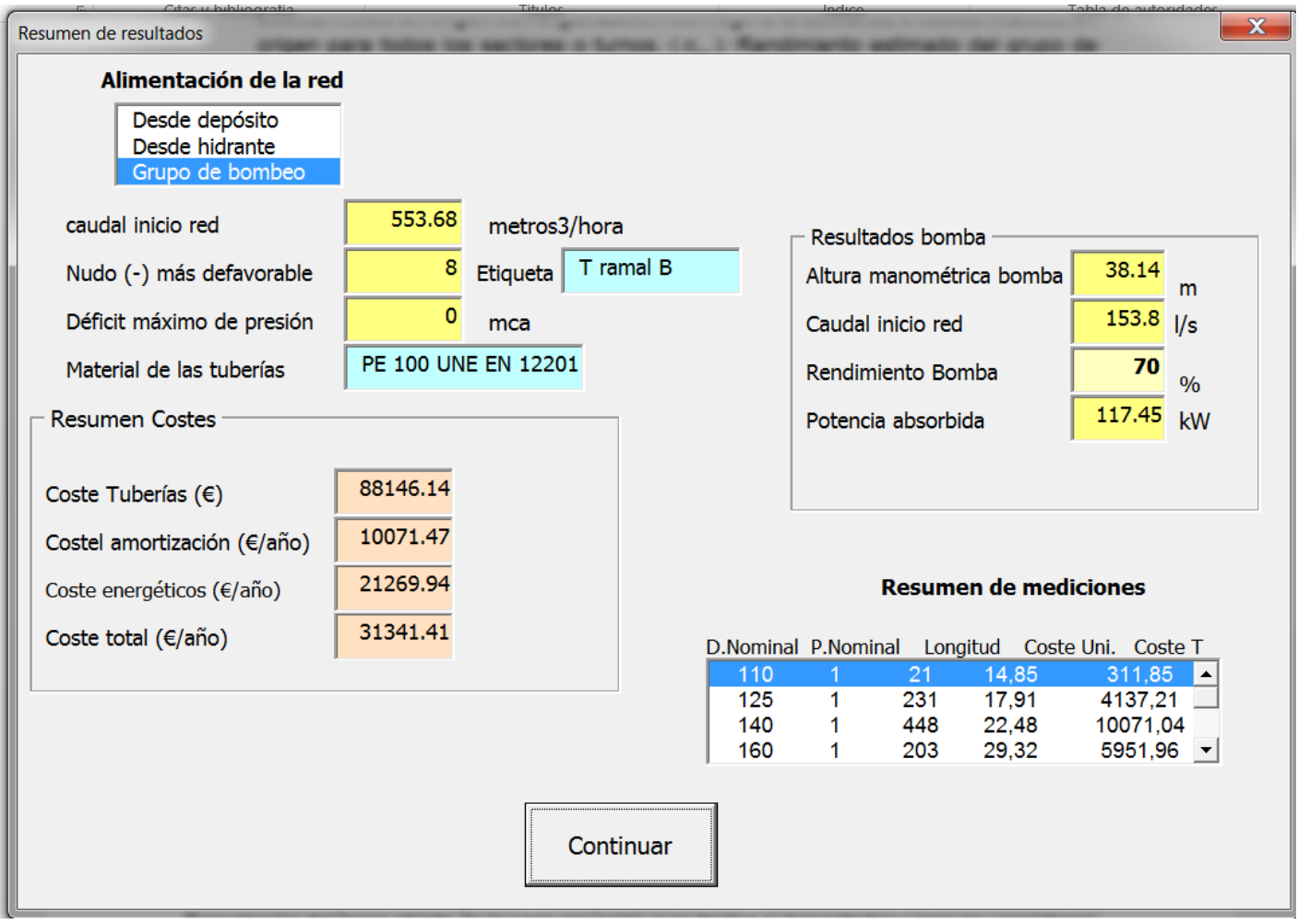

Figura 7: Formulario Resumen de resultados

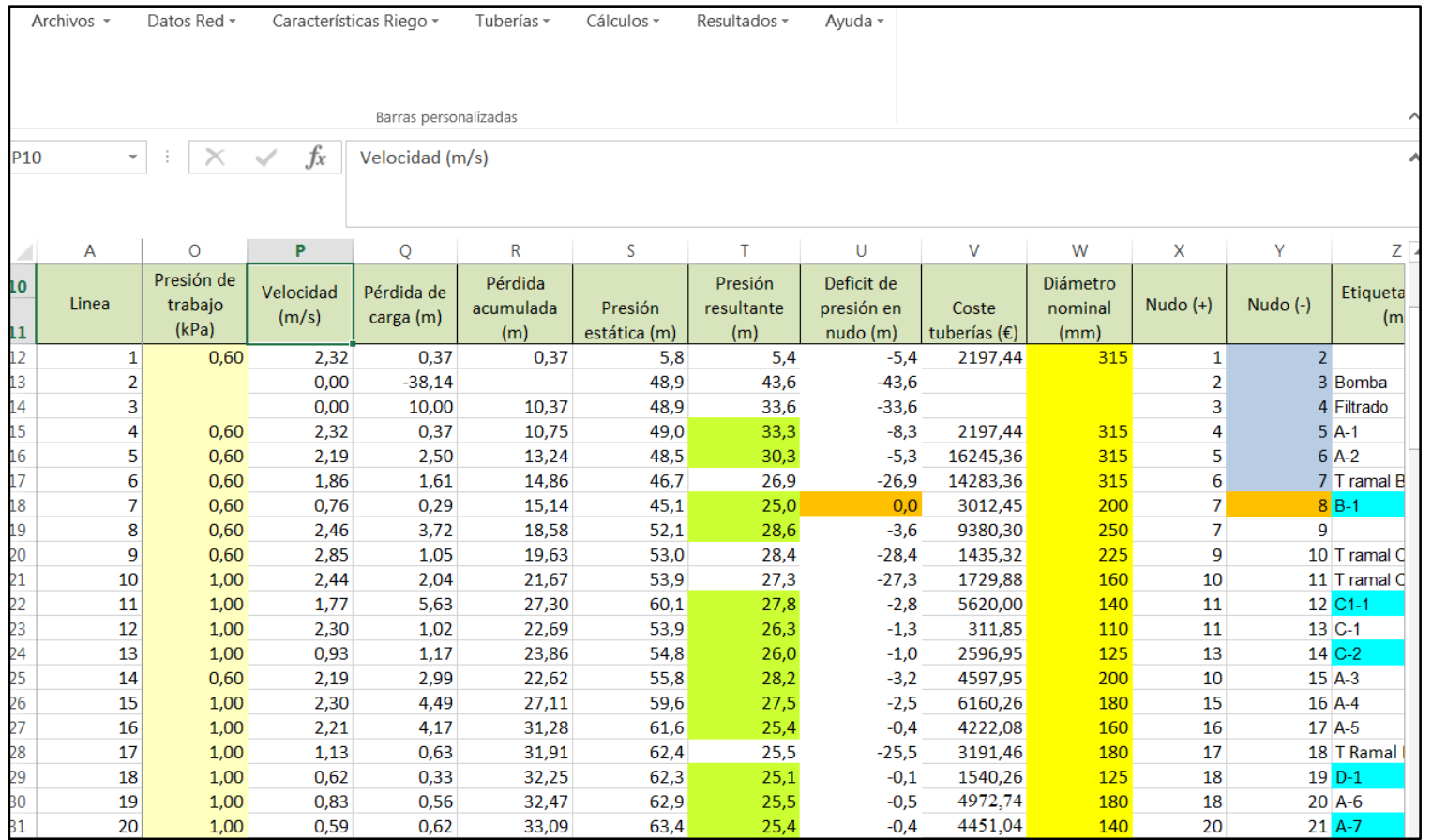

Figura 8: Hoja activa con los resultados del dimensionado

En la Figura 9 aparece el gráfico escalado de la red y los nudos finales de las dos líneas que se visualizan en la parte inferior del formulario aparecen con sendas circunferencias roja y cian. De esta manera se puede recorrer rápidamente todas las líneas de la red y detectar algún error en la conectividad, caudal o diámetro inadecuado o susceptible de ser modificado. 
Desde el menú de resultados (Figura 10) una vez comprobado la bondad de éstos, la aplicación permite crear un nuevo libro donde se da formato a toda la información de la red, datos de partida, resultados y mediciones. Pudiendo ser impreso directamente o copiar como tablas a un documento de texto.

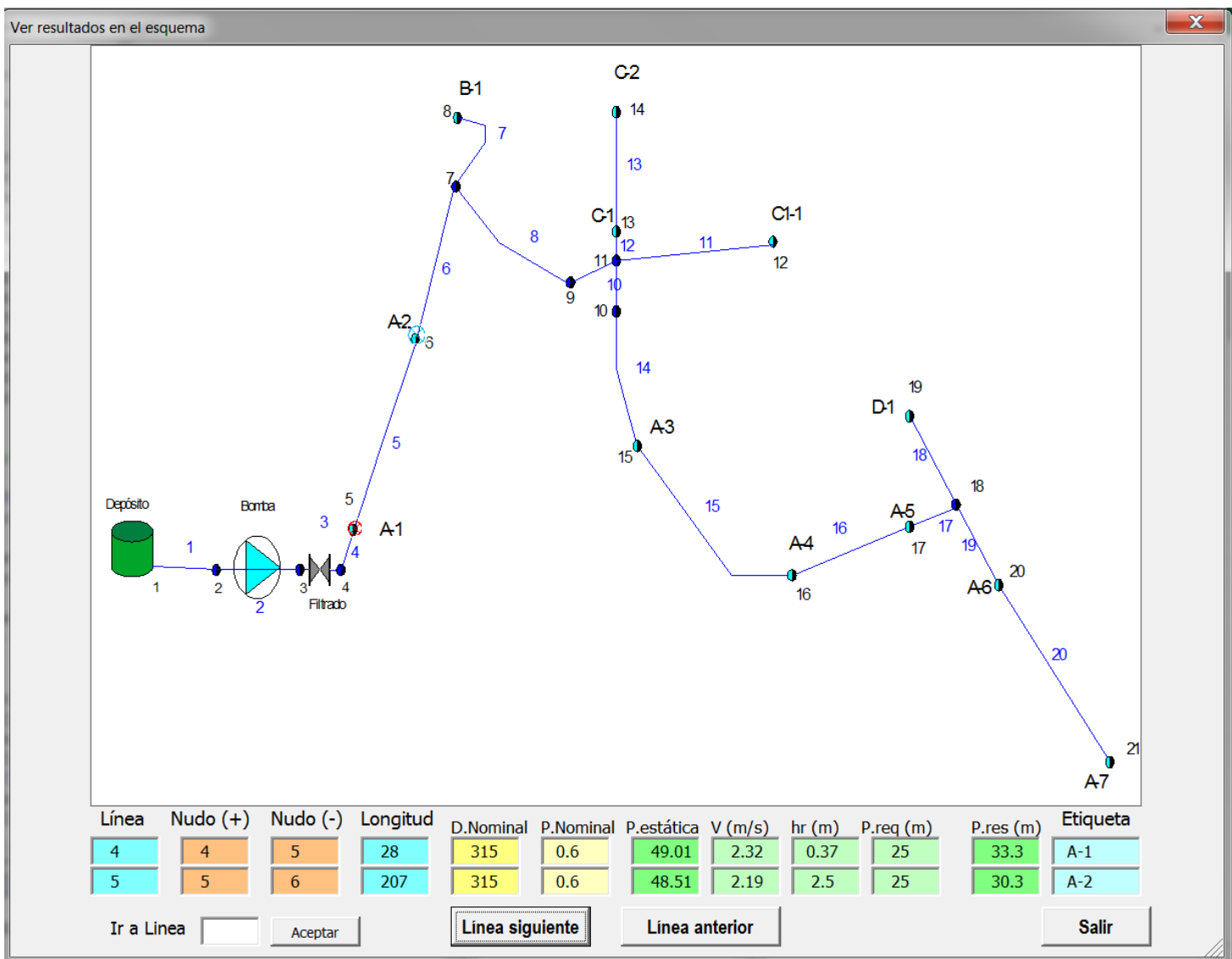

Figura 9: Resultados sobre el esquema gráfico.

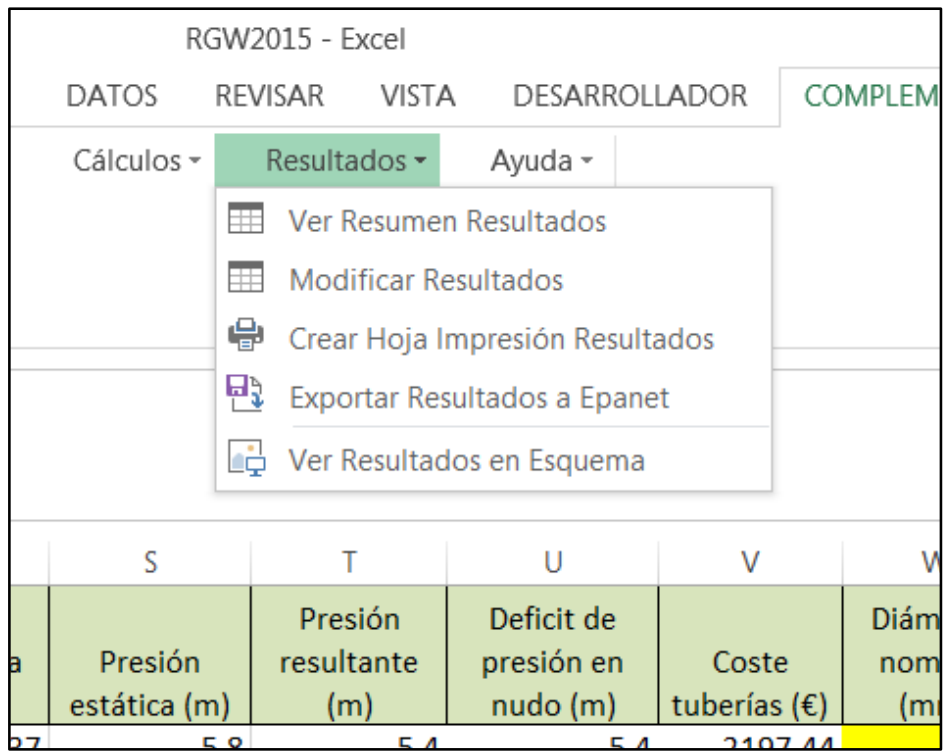

Figura 10: Opciones del menú de resultados. 
Otra opción de interés es la exportación de resultados para su posterior tratamiento con Epanet y validar la respuesta hidráulica de la red dimensionada bajo distintas hipótesis de funcionamiento.

Los diámetros resultantes del dimensionado pueden ser modificados fácilmente por el usuario accediendo a la opción "Modificar Resultados"

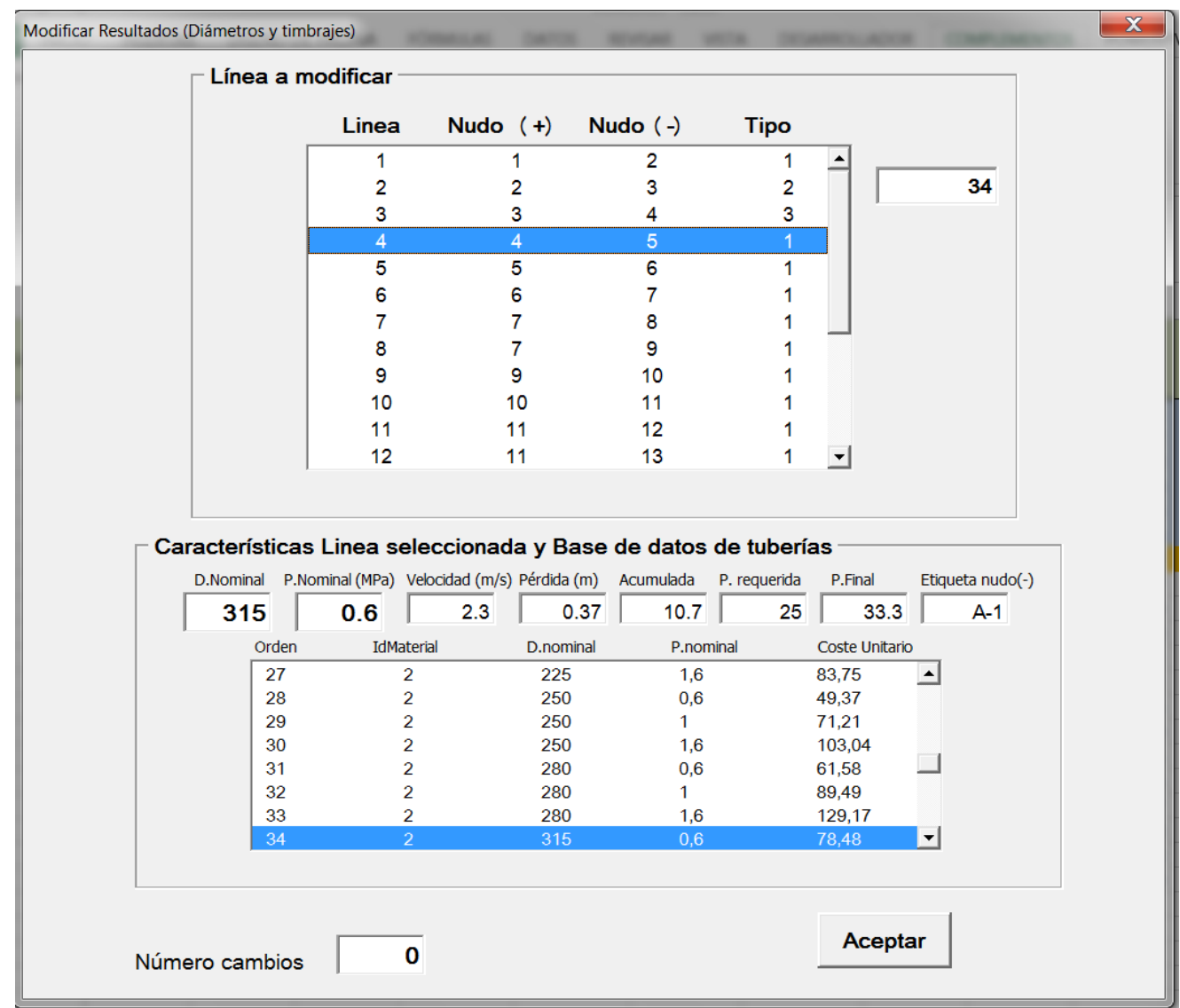

Figura 11: Formulario para la modificación de resultados

\section{Conclusiones}

La aplicación desarrollada, consideramos, resulta de interés tanto en el ámbito académico como profesional siendo de utilidad tanto en el diseño de redes colectivas de riego a presión como para el caso de redes de distribución para grandes superficies.

El entorno utilizado (Excel) facilita la introducción de datos, manejo de la información e interpretación de resultados. Por otra parte al tratarse de una aplicación de difusión libre está abierta a la introducción de continuas mejoras de las que podrán beneficiar tanto los usuarios actuales como futuros.

Se han dimensionado redes de hasta 500 líneas obteniendo resultados satisfactorios y con reducción del coste total frente a versiones anteriores en un 2-5\%. En cualquier caso esta mejora carece de excesiva trascendencia en tanto en cuanto la red debe ser evaluada y analizada mediante la correspondiente aplicación (Epanet) siendo susceptible de modificación de los resultados obtenidos en primera instancia. 
Como mejora inmediata a la aplicación se está desarrollado un entorno gráfico para la introducción de los datos de la red. A su vez los resultados podrán ser exportados a aplicaciones de dibujo técnico (AutoCad o similares)

\section{Bibliografía}

Aliod, R et Al (2011). Nueva herramienta implementada en Gestar 2010 para el dimensionado de tuberías principales en redes de distribución en parcelas y redes de distribución general a turnos. XXX Congreso Nacional de Riegos. Córdoba.

Arviza,J et al. (2007). ASHIDRAR. Un asistente de Cálculo para Problemas de Hidráulica en Riegos a Presión. IV Congreso Nacional de Agrolngeniería (16 p). Albacete (España)

Jiménez Bello, M.A. et Al (2008). Optimización de la sectorización de redes de riego a presión mediante algoritmos genéticos. XXVI Congreso Nacional de Riegos. Huesca

Martínez Alzamora, F \& Arviza, (2002) J. DiopCal: Un módulo de Cálculo de uso general para el Dimensionado Económico de Redes de Riego a Presión. XX Congreso Nacional de Riegos. Ciudad Real

Walkenbach,J. (2010) Excel 2010. Programación con VBA. Editorial Anaya. Madrid 